\title{
The small GTPase RhoA regulates the expression and function of the sodium channel Nav1.5 in breast cancer cells
}

\author{
C. DULONG ${ }^{1 *}$, Y.J. FANG ${ }^{2 *}$, C. GEST ${ }^{1}$, M.H.ZHOU ${ }^{2}$, C. PATTE-MENSAH ${ }^{3}$, A.G. MENSAH-NYAGAN ${ }^{3}$,

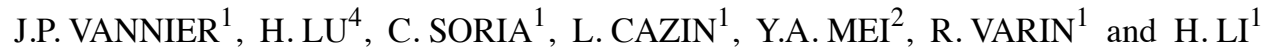 \\ ${ }^{1}$ MERCI, EA 3829, Faculté de Médecine et de Pharmacie, Université de Rouen, Rouen, France; \\ ${ }^{2}$ Center for Brain Science Research, School of Life Science, Fudan University, P.R. China; \\ ${ }^{3}$ Unité de Physiopathologie et Médecine Translationnelle, Faculté de Médecine, Strasbourg; \\ ${ }^{4}$ INSERM UMR-S 728, l'Institut Hématologie, Université Paris 7 Diderot, France
}

Received September 23, 2013; Accepted November 6, 2013

DOI: $10.3892 /$ ijo.2013.2214

\begin{abstract}
Voltage-gated $\mathrm{Na}^{+}$channels (VGSCs) are highly expressed in several types of carcinomas including breast, prostate and lung cancers as well as in mesothelioma and cervical cancers. Although the VGSCs activity is considered crucial for the potentiation of cancer cell migration and invasion, the mechanisms responsible for their functional expression and regulation in cancer cells remain unclear. In the present study, the role of the small GTPase RhoA in the regulation of expression and function of the Nav1.5 channel in the breast cancer cell lines MDA-MB 231 and MCF-7 was investigated. RhoA silencing significantly reduced both Nav1.5 channel expression and sodium current indicating that RhoA exerts a stimulatory effect on the synthesis of an active form of Nav1.5 channel in cancer cells. The inhibition of Nav1.5 expression dramatically reduced both cell invasion and proliferation. In addition, a decrease of RhoA protein levels induced by Nav1.5 silencing was observed. Altogether, these findings revealed: i) the key role of the small GTPase RhoA in upregulation of Nav1.5 channel expression and tumor aggressiveness, and ii) the existence of a positive feedback of Nav1.5 channels on RhoA protein levels.
\end{abstract}

\section{Introduction}

Breast cancer is the most commonly occurring cancer in women. Each year approximately one million of new cases occurred in the world, $25-40 \%$ of patients developing metas-

Correspondence to: Dr H. Li or Dr L. Cazin, Laboratory of MERCI, EA 3829, Faculty of Medicine, University of Rouen, Rouen, France E-mail: li.lu-hong@univ-rouen.fr

E-mail: lionel.cazin@univ-rouen.fr

${ }^{*}$ Contributed equally

Key words: RhoA, voltage-gated $\mathrm{Na}^{+}$channels, Nav1.5 expression, invasion, breast cancer tasis and dying from cancer $(1,2)$. Many problems remain in its clinical management which is generally related to the malignancy types and drug resistance mechanisms as well as metastasis development. Additional investigations to understand the physiopathology of breast cancer are urgently necessary to develop new therapies based on new targets involved in cancer cell invasion and proliferation.

In previous studies we have already demonstrated that the upregulation and activation of the small GTPase RhoA or RhoC contribute to cell invasion leading to breast cancer metastasis $(3,4)$. RhoA signaling pathways are implicated in the activation of FAK, Akt/PI3K, p38MAPK and MLCK, which have been shown to be responsible for cytoskeleton actin reorganization, cell adhesion, motility, migration and invasion (5-9). In many types of cancers, RhoA appears to be overexpressed and/or constitutively activated $(10,11)$, and considered to be a negative clinical prognosis marker $(10,12,13)$.

Recently, it has been shown that the voltage-gated $\mathrm{Na}^{+}$ channels (VGSCs) could be an accelerating factor in malignant cancers (14-17). VGSCs are membrane panning proteins expressed in a wide variety of excitable and non-excitable cells, as well as in many carcinomas including breast cancer $(18,19)$. VGSCs mainly mediate rapid and transient $\mathrm{Na}^{+}$influx into cells and are classically responsible for generation and propagation of action potential. In cancer cells, overexpression of VGSCs and/or their upregulation has been observed. In particular, the Nav1.5 channel type correlates with cancer cell invasion (20-22). Although it seems that the neonatal isoform of Nav1.5 channel may be a potential target for cancer therapy, so far the mechanisms regulating its expression and its functional activity in cancer cells have not been clarified. A previous report revealed that the expression of the neonatal isoform of Nav1.5 was regulated by protein kinase A (23).

In the present study, the Nav1.5 channel expression was analyzed in invasive (MDA-MB-231) or less (MCF-7) invasive breast cancer cell lines. Due to the importance of RhoA in signaling pathways in cancer cell invasion, the involvement of RhoA in the expression and regulation of Nav1.5 was investigated using the real-time RT-PCR and the electrophysiological patch-clamp techniques. 


\section{Materials and methods}

Cell culture. MDA-MB-231 cells were grown in RPMI-1640 medium with $10 \%$ fetal bovine serum (FBS) (Eurobio), $2 \mathrm{mM}$ L-glutamine. MCF-7 cells were grown in H-DMEM medium with $10 \%$ FBS, $4 \mathrm{mM}$ L-glutamine. Both cell lines were obtained from ECACC. All cultures contained $100 \mathrm{IU} / \mathrm{ml}$ penicillin and $100 \mu \mathrm{g} / \mathrm{ml}$ of streptomycin (Eurobio) and were incubated at $37^{\circ} \mathrm{C}$ in a humidified $5 \% \mathrm{CO}_{2}$ atmosphere.

siRNA transfection. Specific siRNAs directed against human RhoA or Nav1.5 was designed using the criteria established by Tuschl. Candidate sequences were compared with cDNA sequences and their specificity verified in the non-redundant human DNA database using a BLAST algorithm [accession through NCBI]. The RhoA siRNA selected was: sense 5'-GAC AUGCUUGCUCAUAGUC-3', antisense 5'-CUGUACGAACG AGUAUCAG-3' and the Nav1.5 siRNA selected was: sense 5'-GGCACAUGAUGGACUUCUU-3', antisense 5'-CCGU GUACCUGAAGAA-3'. Eurogentec negative control siRNA was used as control. siRNAs $(10 \mathrm{nM})$ were introduced into cells by INTERFERin ${ }^{\mathrm{TM}}$-mediated transfection (Ozyme). In patch-clamp experiments, siRNAs were labeled with tetramethyl-rhodamin, and only red cells detected under fluorescent microscope were selected for recording.

Cell proliferation and viability. Cells were seeded $\left(6 \times 10^{3 /}\right.$ well) in 96-well plates in growth medium complemented with 5\% FBS. Cell proliferation/viability was evaluated using a [3-(4,5-dimethylthiazol-2-yl)-5-(3-carboxymethoxyphenyl)2-(4-sulfophenyl)-2H-tetrazolium, inner salt] (MTS, Promega) assay at 24,48 and $72 \mathrm{~h}$ after transfection. Cells were incubated with MTS in culture medium at $37^{\circ} \mathrm{C}$ for $2 \mathrm{~h}$. Optical density was read at $490 \mathrm{~nm}$ using a PowerWave ${ }_{\mathrm{x}}$ spectrophotometer (Bio-Tek Instruments Inc.).

Cancer cell invasion. Cells were cultured for $24 \mathrm{~h}$ in serumfree medium. Cells $\left(7.5 \times 10^{4}\right)$ were seeded in the insert coated with Matrigel. The lower chamber was filled with $0.75 \mathrm{ml}$ of RPMI-1640 containing 10\% FCS to induce chemotaxis. Twenty-four or 72 hours later, the non-migrated cells in the upper chamber were gently scraped away, and invasive cells were fixed with methanol, stained with $1 \%$ toluidine/ $1 \%$ borax solution, and counted with Mercator software (Explora Nova). The invasion by MDA-MB-231 was tested after tranfection with control, RhoA or Nav1.5 siRNAs.

Quantitative polymerase chain reaction $(q P C R)$ assay (realtime RT-PCR). The transfected cells were harvested and total RNA was prepared by SV total RNA isolation system kit (Promega). The purity of total RNA was checked by a ratio of A260/A280 (>1.9). Total RNA (50 ng) was used to synthesize cDNA in $20 \mu 1$ reaction solution using iScript ${ }^{\mathrm{TM}}$ cDNA Synthesis kit (Bio-Rad). Then $2 \mu 1$ of cDNA was used for qPCR assay in duplicates with SYBR Green gene expression assay method. The primers for RhoA (forward primer: 5'-CGC TTTTGGGTACATGGAGT-3', reverse primer: 5'-GAGCAGC TCTCGTAGCCATT-3'), Nav1.5 (forward primer: 5'-CGCCTA CGTGATGAGTGAGA-3', reverse primer: 5'-TAGGAGGG TGGGAAGGAAGT-3') and GAPDH (forward primer: 5'-TGC
ACCACCAACTGCTTAGC-3', reverse primer: 5'-GGCATG GACTGTGGTCATGAG-3') were purchased from Eurogentec, Belgium. The qPCR was performed using Quantifast ${ }^{\mathrm{TM}} \mathrm{SYBR}^{\circledR}$ Green PCR kit (Qiagen) by 10 min of initial denaturation and 44 cycles of $15 \mathrm{sec}$ at $95^{\circ} \mathrm{C}, 60 \mathrm{sec}$ at $60^{\circ} \mathrm{C}$ in a Master Cycler System (Eppendorf). The ratio of interest, $m R N A$ and GAPDH mRNA, was used for analyzing qPCR results.

Western blot analysis. For protein extractions, $2 \times 10^{6}$ cells were seeded into $75-\mathrm{cm}^{2}$ flasks. Forty-eight hours after treatment, proteins were extracted by RIPA buffer complemented with protease and phosphatase inhibitor cocktail, concentration of protein was measured by BCA Protein Assay kit (Pierce). Protein fractions were separated by SDS-PAGE, then transferred onto polyvinylidene difluoride membranes (Amersham) using a dry transfer system (Invitrogen). Membranes were blocked with skim milk, and probed using anti-RhoA (Santa Cruz) and anti-GAPDH (Sigma-Aldrich) primary antibodies. GAPDH is used as control protein and for protein normalization. The detection was done using a secondary peroxidase-conjugated antibody (Dako). After washing the bound antibody was detected with Immobilon western chemiluminescente HRP substrate (Millipore). Then chemiluminescent emission was captured on Kodak XAR film. Images were analyzed by ImageJ software.

Electrophysiology. Whole-cell currents of MDA-MB-231 human breast cancer cells were recorded using a conventional patch-clamp technique. Prior to each experiment, the growth medium was replaced with an external bath solution containing (in $\mathrm{mM}$ ): $\mathrm{NaCl} 144$, KCI 5.4, $\mathrm{MgCl}_{2} 1, \mathrm{CaCl}_{2} 2.5$, D-glucose 5.6, and HEPES 5, adjusted to $\mathrm{pH} 7.3$ with $1 \mathrm{M}$ $\mathrm{NaOH}$. Soft glass patch pipettes of resistance of 5-7 $\mathrm{M} \Omega$ were filled with a solution containing (in $\mathrm{mM}$ ): $\mathrm{NaCl} 5, \mathrm{CsCl} 145$, $\mathrm{MgCl}_{2} 2, \mathrm{CaCl}_{2} 1$, HEPES 10 and EGTA 11, adjusted to pH 7.4 with $1 \mathrm{M} \mathrm{CsOH}$, to block outward $\mathrm{K}^{+}$currents. A holding potential of $-100 \mathrm{mV}$ was applied, unless indicated otherwise. Standard voltage-clamp protocols were used to study the electrophysiological properties of the voltagegated sodium channels on MDA-MB-231 cells. Whole-cell currents of isolated cells were recorded at room temperature $\left(23-25^{\circ} \mathrm{C}\right)$.

Data acquisition and analysis. All current signals were recorded with Axopatch 700B amplifier (Axon Instruments, Union City, CA, USA) in the voltage-clamp mode. Analogue signals were filtered at $3 \mathrm{kHz}$ using a lowpass Bessel filter and series resistance was compensated by $\sim 70 \%$. Currents were corrected on-line for leak and residual capacitance transients by a $\mathrm{P} / 4$ protocol. Electrophysiological signals were sampled at $10 \mathrm{kHz}$ and digitised using Digidata 1440A (Axon Instruments). Data acquisition and analysis of membrane currents were performed with pClamp 10.2 software (Axon Instruments) and/or Origin 8.0 (Origin Lab, Northampton, MA, USA). Values are given as mean \pm SEM with $n$ as the number of cells tested. Statistical analysis was performed using the Student's t-test with paired comparisons where it was relevant. When multiple comparisons were made, data were analyzed by a one-way ANOVA test, followed by Tukey test when significant differences were observed. 
A

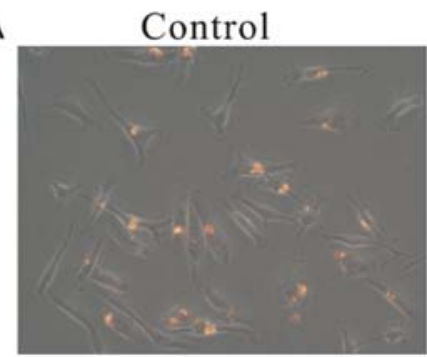

RhoA siRNA

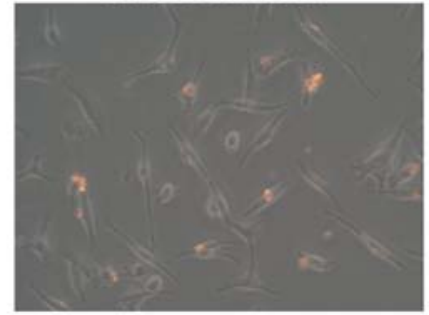

\section{RhoA siRNA}

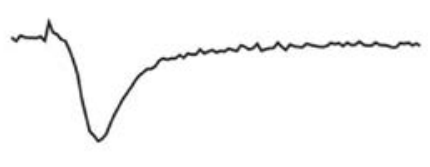

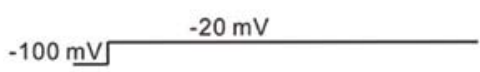

$\frac{1}{1 \mathrm{~ms}}^{0.5 \mathrm{nA}}$

$-100 \mathrm{mV}$

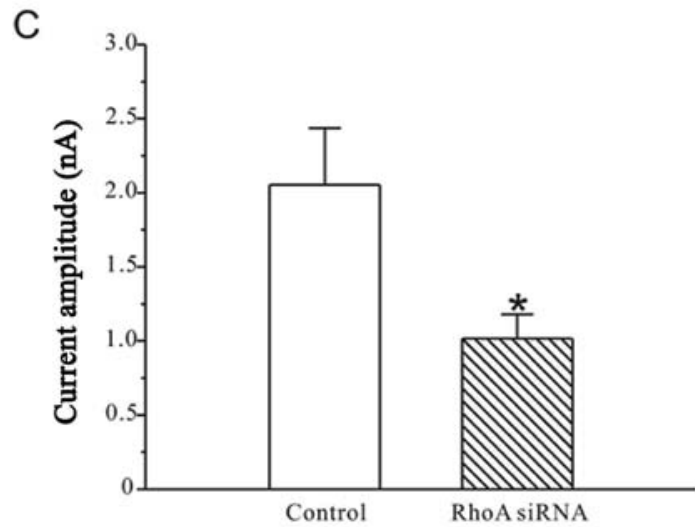

Figure 1. Effect of RhoA siRNAs on the current amplitudes of voltage-gated sodium channels on MDA-MB-231 human breast cancer cells. (A) Merged fluorescence microscope images of cells transfected with blank siRNAs (control) and siRNAs targeting RhoA (RhoA siRNA). Sodium current of cells with red signals indicating successful transfection was recorded by whole-cell patch clamp technique. (B) Typical traces of $I_{\mathrm{Na}}$ elicited by a depolarization potential of $-20 \mathrm{mV}$ on MDA-MB-231 cells before (control) and after (RhoA siRNA) interference with RhoA siRNAs. (C) Statistical analysis of the effect of small interference RNAs targeting RhoA on the amplitude of $I_{\mathrm{Na}}$ on MDA-MB-231 cells. ${ }^{*} \mathrm{p}<0.05$ compared with the corresponding control.

Statistical analysis. The Dunnett test was used for quantitative comparisons between treatments. All experiments were reproduced at least 3 times on different days unless specified otherwise.

\section{Results}

Silencing of RhoA reduces sodium current. Whole-cell patch clamp recordings were used to measure sodium current $\left(I_{\mathrm{Na}}\right)$ in MDA-MB-231 human breast cells. Only the cells with red signals indicating successful transfection were chosen (Fig. 1A). Small interference RNAs targeting RhoA (RhoA siRNAs) dramatically reduced $I_{\mathrm{Na}}$ evoked by depolarizing pulses to $-20 \mathrm{mV}$ from a holding potential of $-100 \mathrm{mV}$. As shown in Fig. 1B and C, the peak current in RhoA siRNAtreated cells $(1016.1 \pm 163.2 \mathrm{pA}, \mathrm{n}=20)$ was decreased by $50.5 \%$ $(\mathrm{p}<0.05)$ compared to the control cells $(2054.7 \pm 380.5 \mathrm{pA}$, $\mathrm{n}=16)$.
In order to clarify whether the decrease of the peak current may result from changes in functional properties or the number of channels expressed on the cell membrane, the effect of RhoA siRNAs on the steady-state activation and inactivation properties of $I_{\mathrm{Na}}$ was further investigated. A protocol of 20-msec depolarizing pulses from a holding potential of $-100 \mathrm{mV}$ to between -60 and $90 \mathrm{mV}$, with $5 \mathrm{mV}$ steps at intervals of $5 \mathrm{sec}$, was used. As shown in Fig. $2 \mathrm{~A}$ and B, the evoked sodium current families were markedly reduced after RhoA silencing. However, RhoA silencing did not significantly modify $(\mathrm{p}<0.05)$ the steady-state activation property of sodium channels. In all treated and untreated cells (16 cells for each group), the half-activation potential was $-35.7 \pm 1.7$ and $-35.9 \pm 1.0$, respectively (Fig. 2C and G).

The effect of RhoA siRNAs on the voltage dependence of steady-state inactivation of sodium channels was studied by inducing $I_{\mathrm{Na}}$ using 1-sec conditioning pre-pulses ranging from -130 to $-10 \mathrm{mV}$, in steps of $5 \mathrm{mV}$ prior to a $-20 \mathrm{mV}$ test 
A

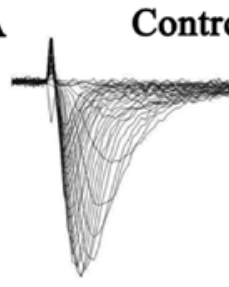

RhoA siRNA

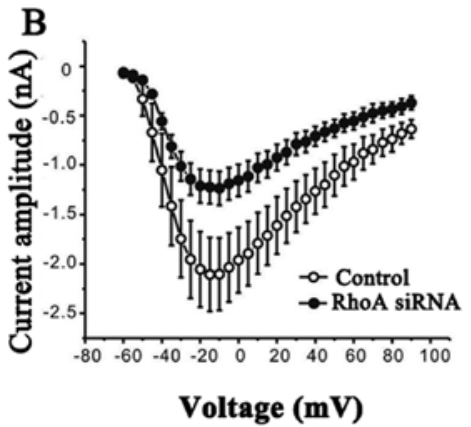

D Control

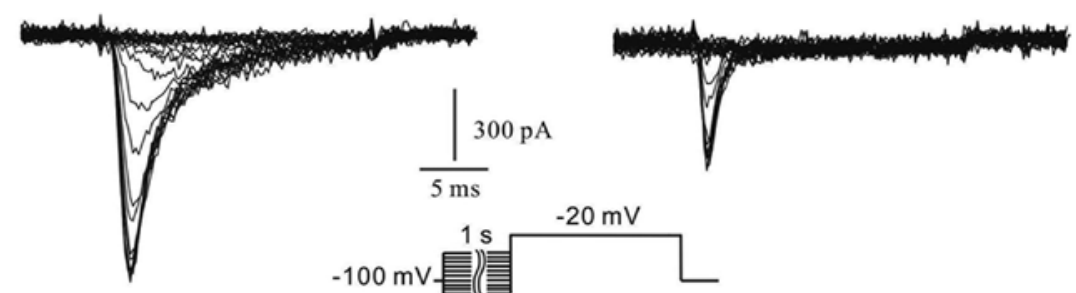

C

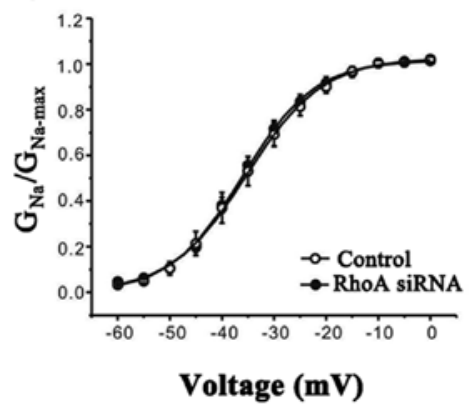

RhoA siRNA

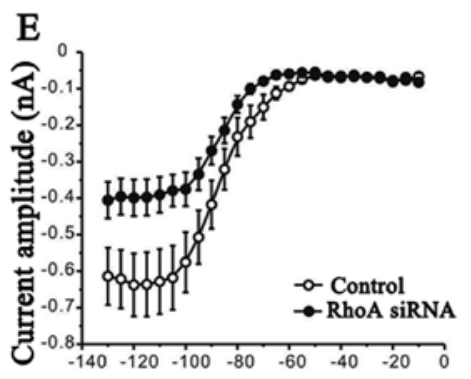

Prepulse voltage (mV)

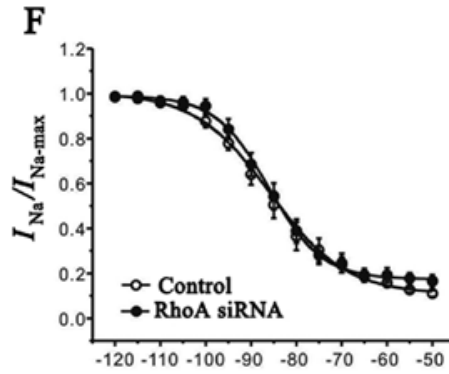

Prepulse voltage $(\mathrm{mV})$

G

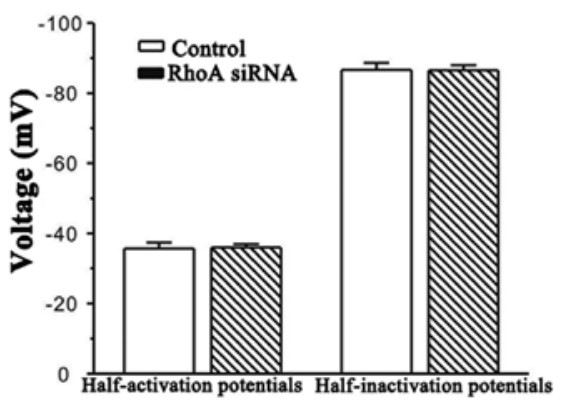

Figure 2. Effects of RhoA siRNAs on the kinetics of voltage-gated sodium channels on MDA-MB-231 human breast cancer cells. (A) Typical traces of the steady-state activation of $I_{\mathrm{Na}}$ before (control) and after (RhoA siRNA) interference with RhoA siRNAs. The cells were held at - $100 \mathrm{mV}$ and depolarized in $5 \mathrm{mV}$ steps from - 60 to $90 \mathrm{mV}$ at intervals of $5 \mathrm{sec}$. (B) The voltage-dependent activation curves of $I_{\mathrm{Na}}$ before (O) and after (•) interference with RhoA siRNAs. The data were obtained from 16 cells for each group and were expressed as mean \pm SEM. (C) Comparison of the plots of normalized conductance as a function of the command potential before $(O)$ and after $(\bullet)$ interference with RhoA siRNAs. RhoA siRNAs did not induce changes in Nav voltage-dependence of activation. (D) Typical traces of the steady-state inactivation of $I_{\mathrm{Na}}$ on MDA-MB-231 cells before (control) and after (RhoA siRNA) interference with RhoA siRNAs. $I_{\mathrm{Na}}$ was elicited using 1-sec conditioning pre-pulses ranging from -130 to $-10 \mathrm{mV}$, in steps of $5 \mathrm{mV}$ prior to a $-20 \mathrm{mV}$ test pulse. (E) The steady-state inactivation curves of $I_{\mathrm{Na}}$ before (O) and after $(\bullet)$ interference with RhoA siRNAs. The data were obtained from 29 cells (13 cells for control and 16 cells for RhoA siRNAs) and were expressed as mean \pm SEM. (F) Comparison of the plots of normalized conductance as a function of the command potential before (O) and after $(\bullet)$ interference with RhoA siRNAs. RhoA siRNAs did not induce changes in the steady-state inactivation of Nav. (G) Statistical analysis of the half-activation and half-inactivation potentials of Nav on MDA-MB-231 cells before and after interference with RhoA siRNAs. 


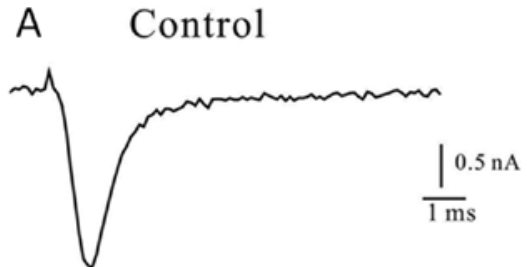

$\mathrm{Na}_{\mathrm{v}} 1.5$ siRNA

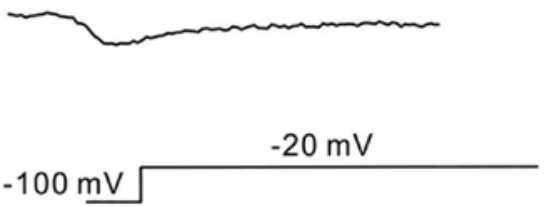

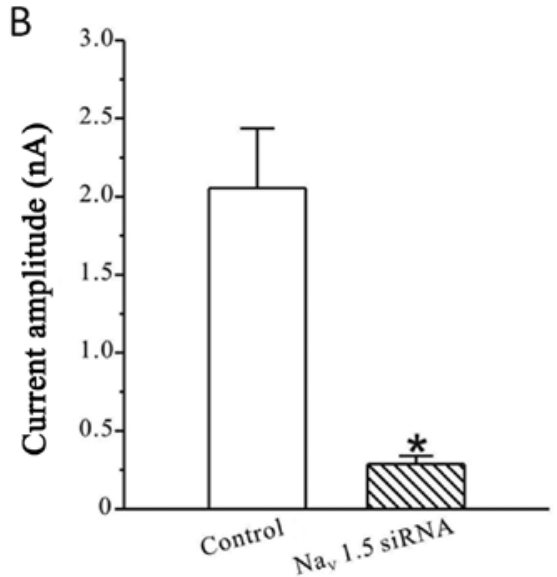

Figure 3. Effect of Nav1.5 siRNAs on the current amplitudes of voltage-gated sodium channels on MDA-MB-231 human breast cancer cells. (A) Typical traces of $I_{\mathrm{Na}}$ elicited by a depolarization potential of $-20 \mathrm{mV}$ on MDA-MB-231 cells before (control) and after interference with Nav1.5 siRNA. (B) Statistical analysis of the effects of small interference RNAs targeting Nav1.5, on the amplitude of $I_{\mathrm{Na}}$ on MDA-MB-231 cells. "p $<0.05$ compared with the corresponding control.
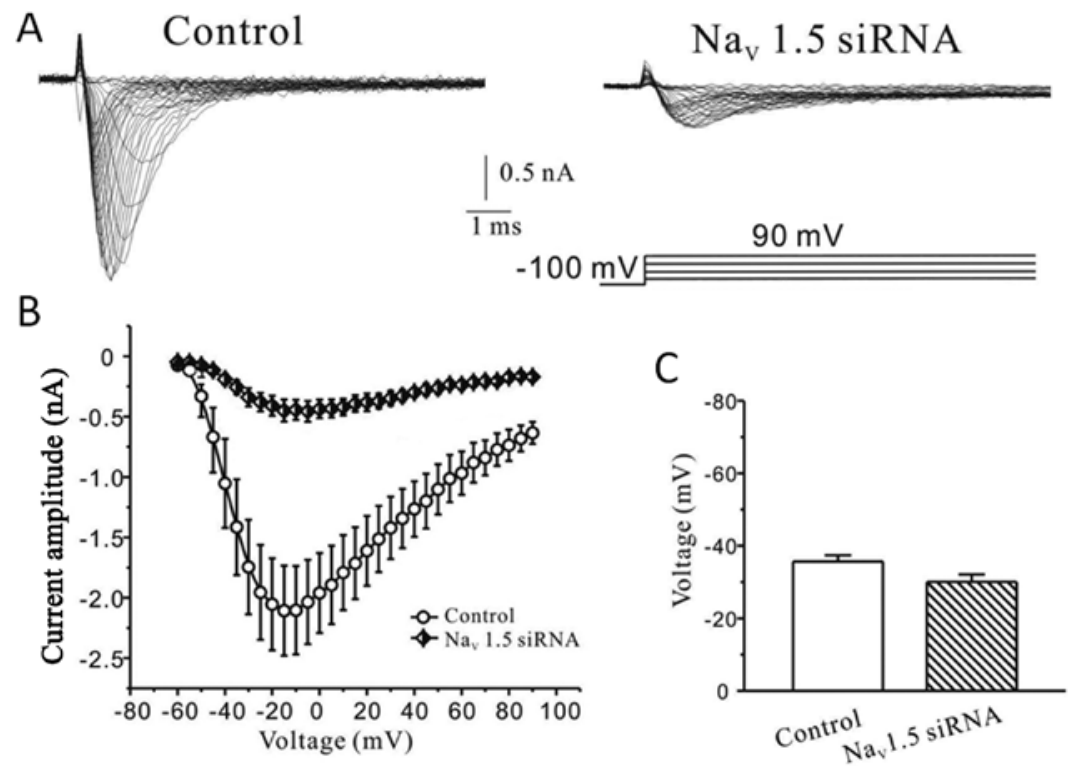

Figure 4. Effects of Nav1.5 siRNA on the kinetics of voltage-gated sodium channels on MDA-MB-231 human breast cancer cells. (A) Typical traces of the steady-state activation of $I_{\mathrm{Na}}$ on MDA-MB-231 cells before (control) and after interference with Nav1.5 siRNA. The cells were held at - $100 \mathrm{mV}$ and depolarized in $5 \mathrm{mV}$ steps from - 60 to $90 \mathrm{mV}$ at intervals of $5 \mathrm{sec}$. (B) Voltage-dependent activation curves of $I_{\mathrm{Na}}$ before and after interference with Nav1.5 siRNA. The data were obtained from 6-7 cells for each group and were expressed as mean \pm SEM. (C) Statistical analysis of the half-activation potential of Nav on MDA-MB-231 cells before and after interference with Nav1.5 siRNA.

pulse. Typical current traces and curves illustrating the relationship between the peak current and the pre-pulse potential is illustrated in Fig. 2D and E. The steady-state inactivation curves were then fitted using the Boltzmann equation of $I_{\mathrm{Na}} /$ $\mathrm{I}_{\mathrm{Namax}}=1 /\left\{1+\exp \left[\left(V_{\mathrm{m}}-V_{\mathrm{m} 1 / 2}\right) / \mathrm{k}\right]\right\}+A$ (Fig. $\left.2 \mathrm{~F}\right)$. In all treated $(\mathrm{n}=16)$ and untreated $(\mathrm{n}=13)$ cells, $V_{\mathrm{m} 1 / 2}$ was $-85.8 \pm 0.6 \mathrm{mV}$ and $-86.1 \pm 0.4 \mathrm{mV}$, respectively without any significant $(\mathrm{p}<0.05)$ changes (Fig. 2G).

Among the different subtypes of VGSCs expressed in MDA-MB-231 human breast cancer cells, the Nav1.5 channel is suggested to be the major functional sodium channel subtype expressed in these cells. To further investigate the expression level of Nav1.5 channels, whole-cell patch clamp recordings were performed in cells treated with the small interference RNAs targeting Nav1.5 channels. As shown in Fig. 3A, transfection of Nav1.5 siRNA strongly reduced the $\mathrm{Na}$ current. Compared to control $(2054.7 \pm 380.5 \mathrm{pA}, \mathrm{n}=16)$, the peak current amplitude in transfected cells was reduced to $288.0 \pm 53.5 \mathrm{pA}(\mathrm{n}=25)$ (Fig. 3B).

We also examined the steady-state activation property of sodium current after interference with Nav1.5 siRNA. Fig. 4A shows representative whole-cell current families recorded from control or Nav1.5 siRNA treated cells. As observed in Fig. 4B the mean I/V curves obtained from control $(n=7)$ or treated $(n=6)$ cells showed that silencing the Nav1.5 sodium channel subtype significantly reduced the sodium peak current elicited by each depolarizing pulse tested. Fig. 4C illustrates that the evoked currents were half-activated at $-30.0 \pm 2.0 \mathrm{mV}$ 
A

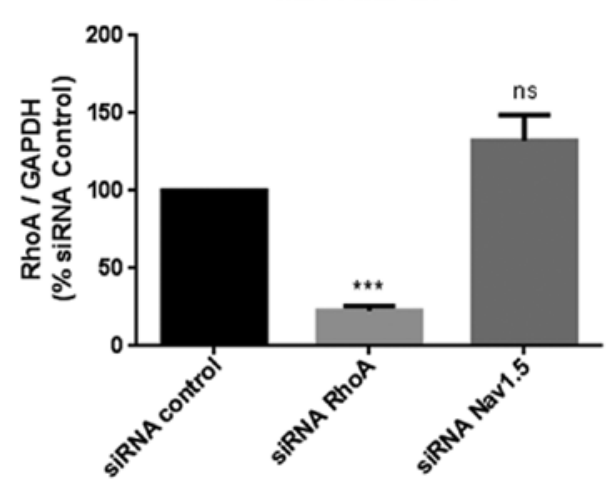

C

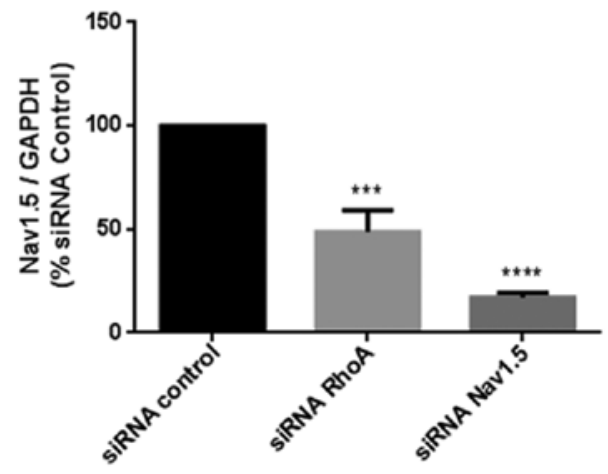

E

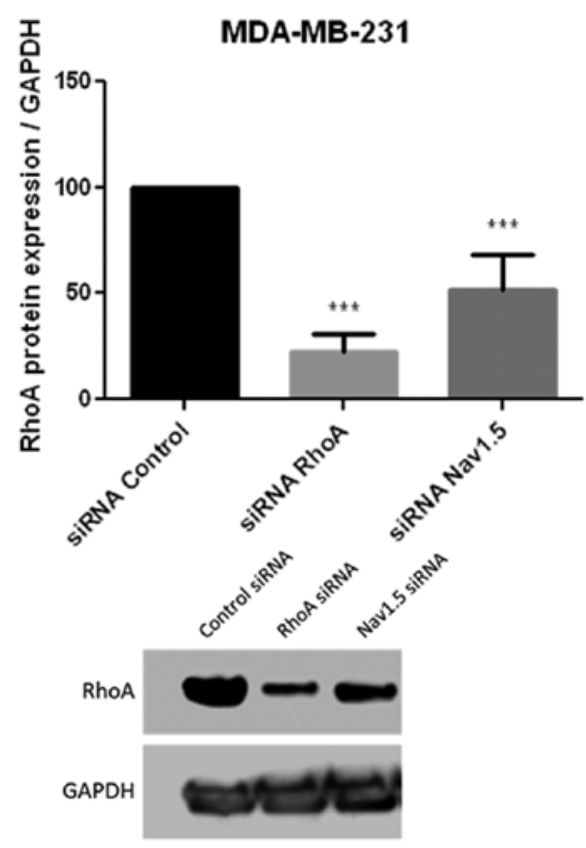

B

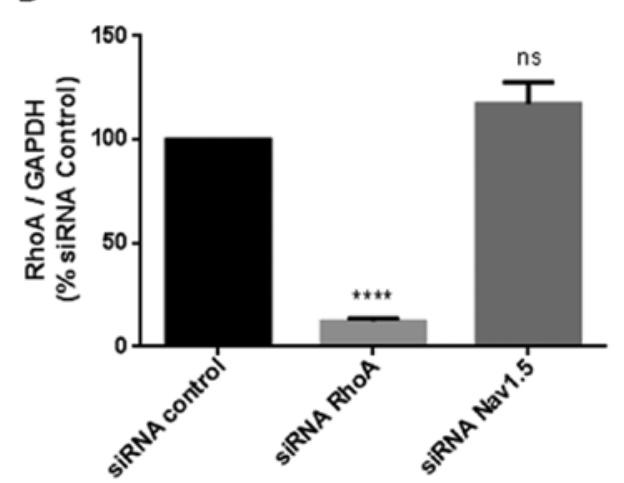

MCF-7

D

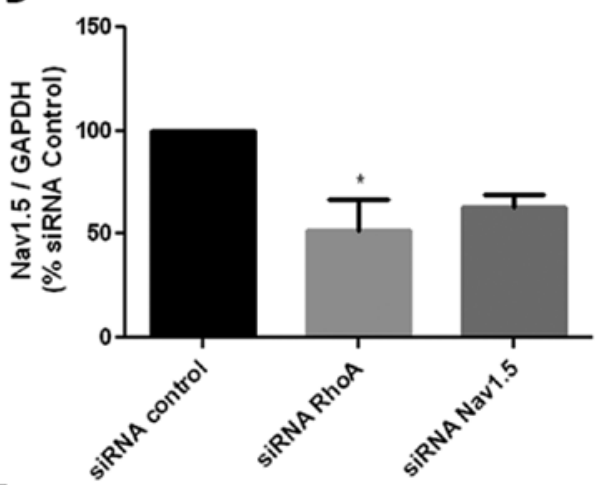

$\mathbf{F}$

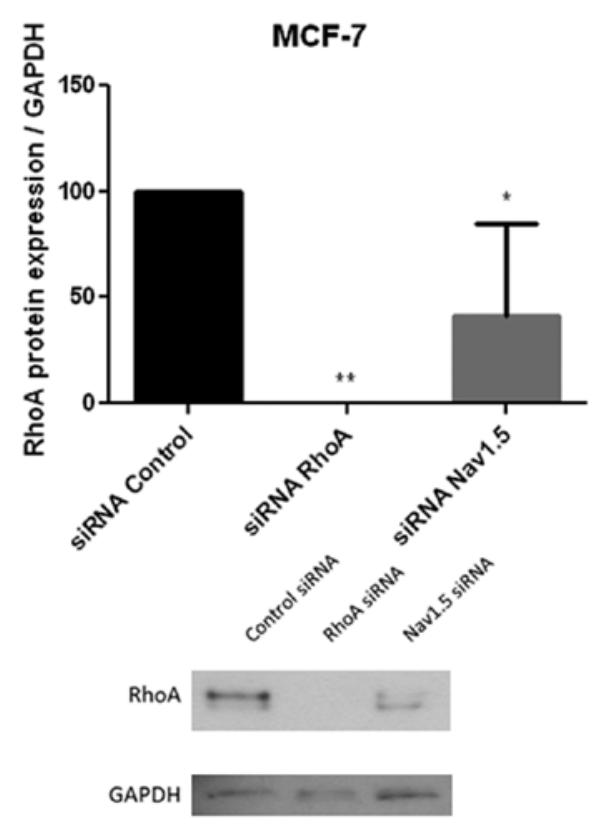

Figure 5. Quantitative detection of RhoA and Nav1.5 expression. (A and B) The effect of RhoA or Nav1.5 siRNAs on RhoA transcription analyzed by real-time RT-PCR (mean \pm SEM, MDA-MB-231: $\mathrm{n}=7$; MCF-7: $\mathrm{n}=5 ;{ }^{* * * *} \mathrm{p}<0.001 ;{ }^{* * * * *} \mathrm{p}<0.0001$ ). (C and D) The effect of RhoA or Nav1.5 siRNAs on Nav1.5 expression. GAPDH expression level was used to control the mRNA concentration used in the experiments (mean \pm SEM, MDA-MB-231: $n=4$; MCF-7: $n=3$; ${ }^{*}<<0.05$, ${ }^{* * * *} \mathrm{p}<0.001,{ }^{* * * * *} \mathrm{p}<0.0001$ ). (E and F) Western blot analysis of RhoA protein level in MDA-MB-231 (mean \pm SEM, $\left.\mathrm{n}=4 ;{ }^{* * *} \mathrm{p}<0.01,{ }^{* * * * *} \mathrm{p}<0.0001\right)$ and MCF-7 (mean \pm SEM, $\mathrm{n}=2$ ) cells, respectively at $48 \mathrm{~h}$ after treatment with RhoA or Nav1.5 siRNAs. The protein concentration of each band was analyzed by imageJ software and are presented as histograms.

after silencing Nav1.5 channels without any significant difference ( $\mathrm{p}>0.05)$ compared to the control $(-35.7 \pm 1.7 \mathrm{mV})$.

Inhibition of RhoA expression downregulates Nav1.5 channels at transcription level. To further investigate the regulation mechanisms of RhoA on Nav1.5 channels, we compared the expression level of the channel molecules in the presence of RhoA or after RhoA silencing. In the present set of experiments, the small interference RNA anti-RhoA or anti-Nav1.5 were used in both aggressive MDA-MB-231 and less aggres- 

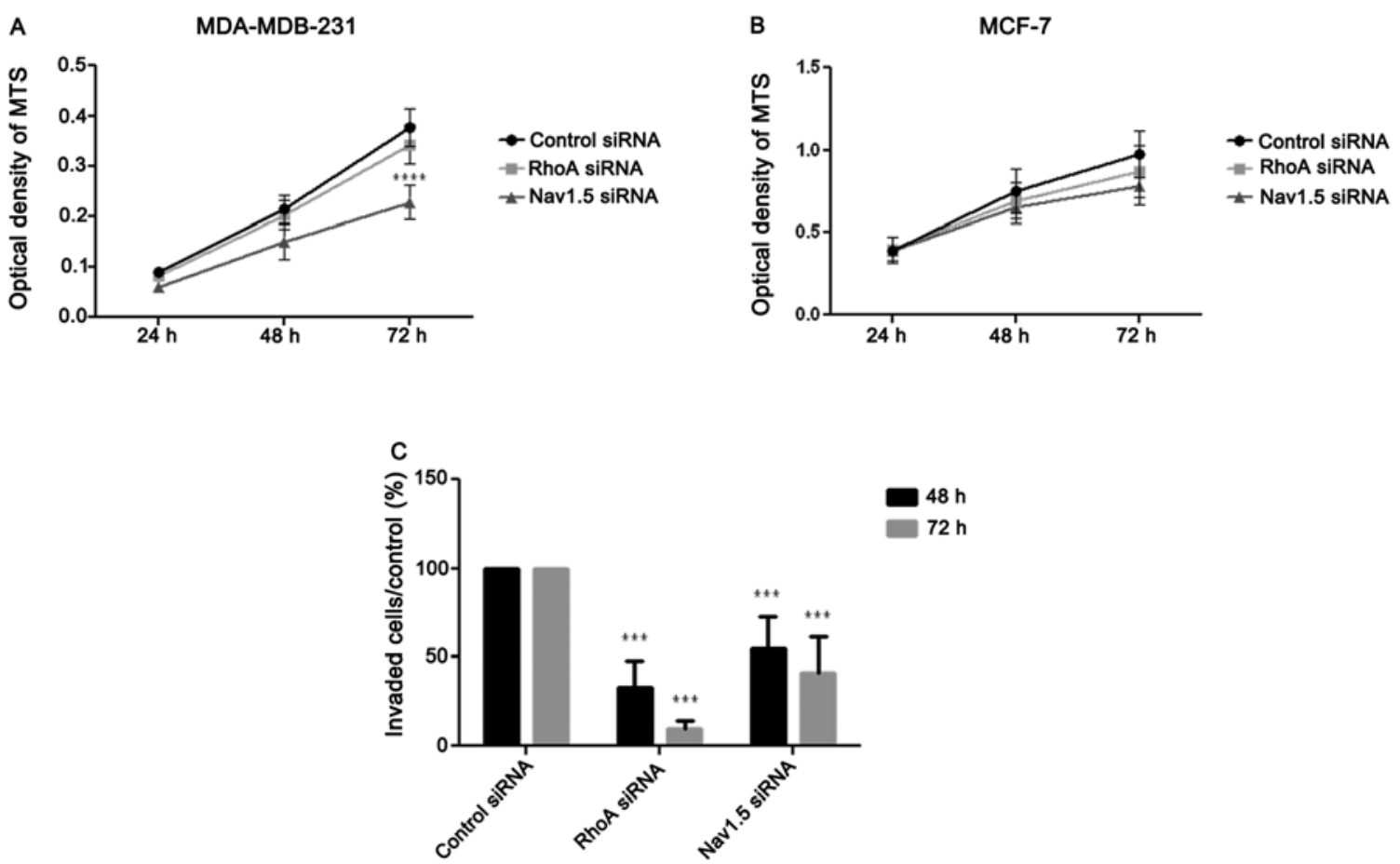

Figure 6. Tumor cell proliferation and invasion analysis. (A and B) Evaluation of cell proliferation after the siRNAs treatment of MDA-MB-231 (mean \pm SEM, $\left.\mathrm{n}=7 ;{ }^{* * * * *} \mathrm{p}<0.0001\right)$ and MCF-7 (mean \pm SEM, $\mathrm{n}=8$ ) cells. Cells were treated with $10 \mathrm{nM}$ siRNAs (control or RhoA or Nav1.5). Cell survival was detected by MTS assay after 24, 48 and $72 \mathrm{~h}$ of treatment. (C) Evaluation of RhoA or Nav1.5 siRNAs on MDA-MB-231 cell invasion in Boyden chamber system. The number of invaded cells was counted $48 \mathrm{~h}\left(\right.$ mean \pm SEM, $\left.\mathrm{n}=6 ;{ }^{* * * *} \mathrm{p}<0.001,{ }^{* * * *} \mathrm{p}<0.0001\right)$ and $72 \mathrm{~h}($ mean $\pm \mathrm{SEM}, \mathrm{n}=4)$ after each treatment.

sive MCF-7 breast cancer cells. Real-time RT-PCR was applied to evaluate the transcriptional level of RhoA or Nav1.5 expression. The histograms of Fig. 5A and $\mathrm{B}$ revealed that siRNA anti-RhoA markedly and significantly inhibited RhoA expression in both types of tumor cells (MDA-MB-231 cells: $\mathrm{p}<0.001, \mathrm{n}=7$; MCF-7 cells: $\mathrm{p}<0.0001, \mathrm{n}=5$ ) while siRNA antiNav1.5 had no effect on RhoA transcriptional level. In contrast, interestingly, as observed in Fig. 5C and D, after siRNA antiRhoA treatment, the transcriptional level of Nav1.5 channels appeared to be significantly decreased (MDA-MB-231 cells: $\mathrm{p}<0.001, \mathrm{n}=4$; MCF-7 cells: $\mathrm{p}<0.05, \mathrm{n}=3$ ) with a similar range of inhibition in both types of breast cancer cells. It has to be noted that the inhibition level of Nav1.5 expression by siRNA anti-Nav1.5 was stronger in MDA-MB-231 than in MCF-7 cells.

Inhibition of Nav1.5 expression downregulates the RhoA protein level. We investigated a potent effect of Nav1.5 channels on RhoA expression. Nav1.5 silencing did not modify the transcriptional level of RhoA (Fig. 5A and B) while it decreased its protein level in both aggressive (MDA-MB-231) and less aggressive (MCF-7) cells (Fig. 5E and F). The western blots shown in Fig. 5 clearly revealed that after a 48-h transfection, siRNA anti-Nav1.5 reduced by $>50 \%$ the RhoA protein level (MDA-MB-231 cells: $p<0.01, n=4$; MCF-7 cells: $n=2$ ).

siRNA anti-Nav1.5 inhibits cell proliferation of $M D A-M B-231$ but not of MCF-7. Additional experiments were conducted on cell proliferation. Results illustrated in Fig. 5A and B indicated that only siRNA anti-Nav1.5 inhibited cell proliferation in MDA-MB-231 cell line ( $<<0.0001 ; n=7$ after 72 -h treatment) while RhoA silencing failed to exert any effect. In contrast, in MCF-7 cells no significant effect was detected after treatment with siRNA anti-RhoA or anti-Nav1.5 $(n=8)$.

RhoA and Nav1.5 promote tumor cell invasion. In order to establish the relationship between RhoA or Nav1.5 and tumor cell invasion, Boyden chamber assays were performed on MDA-MB-231 cells (MCF-7 cells have no invasive activity in this condition) after treatments with siRNA anti-RhoA or anti-Nav1.5. As shown in Fig. 6C, each siRNA tested significantly $(\mathrm{p}<0.0001, \mathrm{n}=6$ after 48 - $\mathrm{h}$ treatment; $\mathrm{p}<0.001, \mathrm{n}=4$ after $72 \mathrm{~h}$ ) inhibited cell invasion. It was noted that the RhoA silencing appeared to have a more pronounced effect than that of Nav1.5.

\section{Discussion}

In previous studies, it was suggested that the upregulation and activation of the small GTPase RhoA contribute to cell invasion leading to metastasis $(10,13)$. Numerous data have elucidated that high RhoA expression and activation levels may be a negative prognostic marker in cancer treatment $(10,12,13)$. We have also demonstrated that in the aggressive breast cancer cells, the proliferation and invasiveness were considerably reduced when the small GTPase RhoA was inhibited $(24,25)$. Thus, we have developed a strategy of molecular therapy using anti-RhoA siRNAs. The efficacy of i.v.-administered encapsulated anti-RhoA siRNA in chitosancoated polyiso-hexylcyanoacrylate (PIHCA) nanoparticles in xenografted aggressive breast cancer MDA-MB-231 was demonstrated $(3,4)$. 
To understand the physiopathology of breast cancer, other reports suggested that upregulation of voltage-gated $\mathrm{Na}^{+}$channels could be an accelerating factor in metastatic disease (26). The Na channel expression level appeared to be closely linked to the metastatic development in human breast cancer (19). As MDA-MB-231 cells are known to highly express Nav1.5 channels on the cytoplasmic membrane, we investigated the relationship between the expression of RhoA and that of $\mathrm{Na}$ channels, particularly the Nav1.5 channels.

The present study was carried out on both invasive (MDA-MB-231) and less-invasive (MCF-7) cells. The expression level of Nav1.5 channels in both cell lines showed the expected results. The expression level appeared to be higher in MDA-MB-231 than in MCF-7 cells, in correlation with RhoA expression and activation pattern.

We found that RhoA controls the expression of Nav1.5 that is recognized as the main type of functional VGSCs. The silencing of RhoA dramatically reduced the expression of Nav1.5 at its transcriptional level. Sustained ( $>48 \mathrm{~h}$ ) treatment with siRNA anti-RhoA markedly depressed sodium current. In addition, we found that RhoA silencing exerted a much stronger inhibitory effect on Nav1.5 than Nav1.7 (data not shown). These results indicated that RhoA plays a key role in sodium current, most probably via modulating the level of Nav1.5 channels. So far, this effect of RhoA on VGSC expression and function has not been reported in cancer cells or in non-tumor cells. Investigations in different types of normal and tumor cells remain to be done to clarify whether this effect of RhoA corresponds to physiological ubiquitous or tumor specific mechanism. Since RhoA is implicated in the formation of actin fibers, it would be of interest to know whether the suppression of RhoA by siRNA may lead to an interrupted integration of sodium channels into the cell membrane.

We noted that anti-Nav1.5 siRNA, not only markedly decreased sodium current by $70 \%$, but also inhibited cell invasion and migration by $60-80 \%$. Such results strongly support the notion that Nav1.5 is the main VGSC implicated in MDA-MB-231 cell invasiveness as suggested previously by Brackenbury et al and Gao et al $(20,27)$.

Interestingly, a positive feed-back of Nav1.5 on RhoA was observed in this study. We found that inhibition by antiNav1.5 siRNA led to a decrease in RhoA protein level in the cells. Surprisingly, the result of RT-PCR showed no change in the RhoA mRNA level. It suggests that Nav1.5 might affect the stability of the RhoA protein. Many post-transcriptional and post-translational events occur in cells such as phosphorylation, transglutamination, palmitoylation, AMPylation, isoprenylation, and ubiquitylation, and these modifications determine the distribution and life cycle of RhoA $(28,29)$. Described mechanisms governing the stability of Rho proteins include phosphorylation of serine 188 on RhoA that protect against ubiquitin-mediated proteasomal degradation and geranylgeranylation which facilitates proteasomal degradation of Rho (30,31).

Nav1.5-regulated RhoA protein level might be partially explained by a regulatory mechanism which involves sodium currents and intracellular calcium gradient. As a rule, Nav1.5mediated sodium currents influence the electrochemical gradient of calcium because sodium influx activates voltagedependent calcium channels and induces calcium entry.
Indeed, the RhoA level was regulated by cytosolic calcium concentration. Rao et al demonstrated that the reduction of calcium concentration led to a decrease in Rho expression and the decrease in cytosolic calcium markedly destabilized the RhoA protein and accelerates its degradation (32). This allows assuming a likely accelerated RhoA degradation when blocking Nav1.5 function.

Therefore, a positive feed-back between RhoA and VGSCs would represent a crucial mechanism in oncogenesis. As RhoA is overexpressed and constitutively activated in various cancers including breast cancer $(10,11)$, RhoA-mediated cancer invasiveness and metastasis could be partially via VGSC upregulation. Effectively it has been shown that in prostate cancer cells a significant increase in voltage-dependent channels was observed and found relative to the stimulation by EGF, a growth factor known for activating RhoA (33). Therefore, targeting RhoA signaling may be an interesting approach in cancer therapy.

Recently, the dominant VGSC was found to be an embryonic/neonatal splice variant (nNav1.5) consistent with the gene expression being 'oncofoetal'. The molecular differences between the adult and neonatal isoforms of the VGSC/Nav1.5 are 31 nucleotide differences, resulting in 7 amino acid differences (26). It was proposed that $\mathrm{nNav1.5}$ is a novel marker with significant clinical potential for management of metastatic breast cancer. However, the mechanisms responsible for the expression of VGSC especially the neonatal isoforms in metastatic cancers are not well elucidated. Therefore, further investigation of $\mathrm{nNav1.5}$ expression and $\mathrm{RhoA}$ regulation seems to be of interest to better understand the regulation mechanisms between RhoA signaling and VGSC function and their implication in cancer malignancy.

\section{Acknowledgements}

C. Dulong was surpported finacially by La Région Normandie. Grant sponsor: Groupement des Entreprises Francaises dans la Lutte contre le Cancer de Rouen (GEFLUC of Rouen), the Ligue Regionale de Haute Normandie de Lutte contre le Cancer, Association Ti'Toine. We thank Catherine Bouquet and Elisabeth Legrand for their technical assistance.

\section{References}

1. Guarneri V and Conte P: Metastatic breast cancer: therapeutic options according to molecular subtypes and prior adjuvant therapy. Oncologist 14: 645-656, 2009.

2. Decensi A, Dunn BK, Puntoni M, Gennari A and Ford LG: Exemestane for breast cancer prevention: a critical shift? Cancer Discov 2: 25-40, 2012

3. Pillé JY, Denoyelle C, Varet J, Bertrand JR, Soria J, Opolon P, Lu H, Pritchard LL, Vannier JP, Malvy C, Soria C and Li H: Anti-RhoA and anti-RhoC siRNAs inhibit the proliferation and invasiveness of MDA-MB-231 breast cancer cells in vitro and in vivo. Mol Ther 11: 267-274, 2005.

4. Pillé JY, Li H, Blot E, Bertrand JR, Pritchard LL, Opolon P, Maksimenko A, Lu H, Vannier JP, Soria J, Malvy C and Soria C: Intravenous delivery of anti-RhoA small interfering RNA loaded in nanoparticles of chitosan in mice: safety and efficacy in xenografted aggressive breast cancer. Hum Gene Ther 17: 1019-1026, 2006.

5. Nobes CD and Hall A: Rho, rac, and cdc42 GTPases regulate the assembly of multimolecular focal complexes associated with actin stress fibers, lamellipodia, and filopodia. Cell 81: 53-62, 1995. 
6. Schmitz AA, Govek EE, Böttner B and Van Aelst L: Rho GTPases: signaling, migration, and invasion. Exp Cell Res 261: $1-12,2000$.

7. Del Re DP, Miyamoto S and Brown JH: Focal adhesion kinase as a RhoA-activable signaling scaffold mediating Akt activation and cardiomyocyte protection. J Biol Chem 283: 35622-35629, 2008.

8. Cardone RA, Bagorda A, Bellizzi A, Busco G, Guerra L, Paradiso A, Casavola V, Zaccolo M and Reshkin SJ: Protein kinase A gating of a pseudopodial-located RhoA/ROCK/p38/ NHE1 signal module regulates invasion in breast cancer cell lines. Mol Biol Cell 16: 3117-3127, 2005.

9. Gutjahr MC, Rossy J and Niggli V: Role of Rho, Rac, and Rho-kinase in phosphorylation of myosin light chain, development of polarity, and spontaneous migration of Walker 256 carcinosarcoma cells. Exp Cell Res 308: 422-438, 2005.

10. Gou L, Wang W, Tong A, Yao Y, Zhou Y, Yi C and Yang J: Proteomic identification of RhoA as a potential biomarker for proliferation and metastasis in hepatocellular carcinoma. J Mol Med 89: 817-827, 2011.

11. Leve F and Morgado-Díaz JA: Rho GTPase signaling in the development of colorectal cancer. J Cell Biochem 113: 2549-2559, 2012.

12. Horiuchi A, Kikuchi N, Osada R, Wang C, Hayashi A, Nikaido T and Konishi I: Overexpression of RhoA enhances peritoneal dissemination: RhoA suppression with Lovastatin may be useful for ovarian cancer. Cancer Sci 99: 2532-2539, 2008.

13. Liu Y, Wang Y, Zhang Y, Miao Y, Zhao Y, Zhang PX, Jiang GY, Zhang JY, Han Y, Lin XY, Yang LH, Li QC, Zhao C and Wang EH: Abnormal expression of p120-catenin, E-cadherin, and small GTPases is significantly associated with malignant phenotype of human lung cancer. Lung Cancer 63: 375-382, 2009.

14. Balasuriya D, Stewart AP, Crottès D, Borgese F, Soriani O and Edwardson JM: The sigma-1 receptor binds to the Nav1.5 voltage-gated $\mathrm{Na}^{+}$channel with 4 -fold symmetry. J Biol Chem 287: 37021-37029, 2012

15. Gillet L, Roger S, Besson P, Lecaille F, Gore J, Bougnoux P, Lalmanach G and Le Guennec JY: Voltage-gated sodium channel activity promotes cysteine cathepsin-dependent invasiveness and colony growth of human cancer cells. J Biol Chem 284: 8680-8691, 2009

16. House CD, Vaske CJ, Schwartz AM, Obias V, Frank B, Luu T, Sarvazyan N, Irby R, Strausberg RL, Hales TG, Stuart JM and Lee $\mathrm{NH}$ : Voltage-gated $\mathrm{Na}^{+}$channel $\mathrm{SCN} 5 \mathrm{~A}$ is a key regulator of a gene transcriptional network that controls colon cancer invasion. Cancer Res 70: 6957-6967, 2010.

17. Hernandez-Plata E, Ortiz CS, Marquina-Castillo B, MedinaMartinez I, Alfaro A, Berumen J, Rivera M and Gomora JC: Overexpression of $\mathrm{NaV} 1.6$ channels is associated with the invasion capacity of human cervical cancer. Int J Cancer 130 2013-2023, 2012.

18. Diss JK, Fraser SP and Djamgoz MB: Voltage-gated $\mathrm{Na}^{+}$channels: multiplicity of expression, plasticity, functional implications and pathophysiological aspects. Eur Biophys J 33: 180-193, 2004.

19. Fraser SP, Diss JK, Chioni AM, Mycielska ME, Pan H, Yamaci RF, Pani F, Siwy Z, Krasowska M, Grzywna Z, Brackenbury WJ, Theodorou D, Koyutürk M, Kaya H, Battaloglu E, De Bella MT, Slade MJ, Tolhurst R, Palmieri C, Jiang J, Latchman DS, Coombes RC and Djamgoz MB: Voltage-gated sodium channel expression and potentiation of human breast cancer metastasis. Clin Cancer Res 11: 5381-5389, 2005.
20. Brackenbury WJ, Chioni AM, Diss JK and Djamgoz MB: The neonatal splice variant of Nav1.5 potentiates in vitro invasive behaviour of MDA-MB-231 human breast cancer cells. Breast Cancer Res Treat 101: 149-160, 2007.

21. Brisson L, Gillet L, Calaghan S, Besson P, Le Guennec JY, Roger $\mathrm{S}$ and Gore $\mathrm{J}$ : $\mathrm{Na}(\mathrm{V}) 1.5$ enhances breast cancer cell invasiveness by increasing NHE1-dependent $\mathrm{H}(+)$ efflux in caveolae. Oncogene 30: 2070-2076, 2011.

22. Yang M, Kozminski DJ, Wold LA, Modak R, Calhoun JD, Isom LL and Brackenbury WJ: Therapeutic potential for phenytoin: targeting $\mathrm{Na}(\mathrm{v}) 1.5$ sodium channels to reduce migration and invasion in metastatic breast cancer. Breast Cancer Res Treat 134: 603-615, 2012.

23. Chioni AM, Shao D, Grose R and Djamgoz MB: Protein kinase A and regulation of neonatal Nav1.5 expression in human breast cancer cells: activity-dependent positive feedback and cellular migration. Int J Biochem Cell Biol 42: 346-358, 2010.

24. Denoyelle C, Vasse M, Körner M, Mishal Z, Ganné F, Vannier JP, Soria $\mathrm{J}$ and Soria C: Cerivastatin, an inhibitor of HMG-CoA reductase, inhibits the signaling pathways involved in the invasiveness and metastatic properties of highly invasive breast cancer cell lines: an in vitro study. Carcinogenesis 22: 1139-1148, 2001.

25. Vincent L, Chen W, Hong L, Mirshahi F, Mishal Z, MirshahiKhorassani T, Vannier JP, Soria J and Soria C: Inhibition of endothelial cell migration by cerivastatin, an HMG-CoA reductase inhibitor: contribution to its anti-angiogenic effect. FEBS Lett 495: 159-166, 2001

26. Onkal R and Djamgoz MB: Molecular pharmacology of voltagegated sodium channel expression in metastatic disease: clinical potential of neonatal Nav1.5 in breast cancer. Eur J Pharmacol 625: 206-219, 2009.

27. Gao R, Wang J, Shen Y, Lei M and Wang Z: Functional expression of voltage-gated sodium channels Nav1.5 in human breast cancer cell line MDA-MB-231. J Huazhong Univ Sci Technolog Med Sci 29: 64-67, 2009.

28. Liu M, Bi F, Zhou X and Zheng Y: Rho GTPase regulation by miRNAs and covalent modifications. Trends Cell Biol 22: 365-373, 2012.

29. David M, Petit D and Bertoglio J: Cell cycle regulation of Rho signaling pathways. Cell Cycle 11: 3003-3010, 2012.

30. Rolli-Derkinderen M, Sauzeau V, Boyer L, Lemichez E, Baron C, Henrion D, Loirand G and Pacaud P: Phosphorylation of serine 188 protects RhoA from ubiquitin/proteasome-mediated degradation in vascular smooth muscle cells. Circ Res 96: 1152-1160, 2005.

31. Von Zee CL and Stubbs EB Jr: Geranylgeranylation facilitates proteasomal degradation of rho G-proteins in human trabecular meshwork cells. Invest Ophthalmol Vis Sci 52: 1676-1683, 2011.

32. Rao JN, Li L, Golovina VA, Platoshyn O, Strauch ED, Yuan JX and Wang JY: $\mathrm{Ca}^{2+}-\mathrm{RhoA}$ signaling pathway required for polyamine-dependent intestinal epithelial cell migration. Am J Physiol Cell Physiol 280: 993-1007, 2001.

33. Uysal-Onganer P and Djamgoz MB: Epidermal growth factor potentiates in vitro metastatic behaviour of human prostate cancer PC-3M cells: involvement of voltage-gated sodium channel. Mol Cancer 6: 76, 2007. 